\title{
PHOTOSPHERIC AND CIRCUMSTELLAR VARIABILITY OF THE RAPIDLY ROTATING O STAR HD 93521
}

\author{
A. W. FULLERTON \\ Bartol Research Institute, University of Delaware, Newark, DE 19716-4793 U.S.A. \\ D. R. GIES \\ Dept. of Physics \& Astronomy, Georgia State University, Atlanta, GA 30303 U.S.A. \\ and \\ C. T. BOLTON \\ David Dunlap Observatory, P.O. Box 360, Richmond Hill, Ont. CANADA L\&C \&YG
}

\section{HD 93521: Pulsation, Rotation, and Mass Loss}

HD 93521 is an $09.5 \mathrm{~V}$ star with several well-documented peculiarities, including: an extremely large projected rotational velocity $(400 \mathrm{~km} / \mathrm{s}$ according to Conti \& Ebbets 1977); absorption line profile variations (Fullerton 1990); weak emission at $\mathrm{H} \alpha$ (Irvine 1989); evidence for a rotationallymodified stellar wind in its UV resonance lines (Massa 1992; Howarth \& Reid 1993); and stellar wind variability in the form of discrete absorption components (Prinja \& Howarth 1986). Thus, HD 93521 provides an ideal laboratory for studying the interaction between rapid rotation and variability in both photospheric layers and the circumstellar environment.

\section{Photospheric Variability}

We obtained a time series of 36 high S/N (350), moderate resolution (7000) spectra of the He II $\lambda 4686$ and He I $\lambda 4713$ lines of HD 93521 with the coudé spectrograph of the $2.1 \mathrm{~m}$ telescope at the McDonald Observatory on 2 nights in 1987 March. These observations show recurrent, regularly spaced "bumps" with semi-amplitude $\sim 1 \%$ of the continuum that traverse the He I line profile from blue to red every 4.8 hours. Analysis of these variations by means of the periodogram technique developed by Gies \& Kullavanijaya (1988) indicates that a single sinusoidal frequency of $13.65 \pm 0.04 \mathrm{~d}^{-1}$ (i.e., period $1.76 \pm 0.01$ hours) extends across the He I $\lambda 4713$ profile. In contrast, similar variations are barely visible in the $\mathrm{He}$ II line, and we could not detect the 1.76-hour period in our observations of it. Simultaneous photometry did not indicate statistically significant light variations with amplitude greater than 0.002 magnitudes in the Strömgren $y$ filter.

The simplest interpretation of the variability in He I $\lambda 4713$ is in terms of a single mode of nonradial pulsation (NRP), with period in the observer's 
frame of 1.76 hours. The variation of pulsation phase with position in the line profile indicates that a high-degree, prograde mode is excited ( $m=$ $-9 \pm 1)$, while the large amplitude suggests that the mode is sectorial $(\ell=$ $|m|)$. Photometric variations are not expected from such a mode because of the nearly perfect cancellation of temperature variations and surface-area distortions between adjacent sectors of the visible disk of the star. Since the NRP amplitude is greatly reduced in He II $\lambda 4686$, we infer that this line must be formed either farther from the photosphere than He I $\lambda 4713$, or closer to the hot, polar caps of the rotationally distorted stellar surface.

\section{Circumstellar Variability}

At various epochs we have also obtained less intensive time series observations of the He I $\lambda 5876$ and $\mathrm{H} \alpha$ lines of HD 93521, both of which are diagnostics of the circumstellar environment. The cores of these lines are blue shifted by $\sim 50 \mathrm{~km} / \mathrm{s}$, and both exhibit small blue- and red-emission peaks that are displaced by $\sim 500 \mathrm{~km} / \mathrm{s}$ from line center. Although the temporal sampling of our data is fragmentary, changes in the shape, strength, and emission $V / R$ ratio are evident from night to night. We cannot determine if the circumstellar variations are cyclical from the present data.

The morphology of these profiles confirms that a weak disk persists around HD 93521, as had been deduced previously from the peculiar UV resonance line profiles of this star (Massa 1992; Howarth \& Reid 1993). The blue shift of the line centers and the most common $V / R$ asymmetries suggest that material is flowing slowly outward through disk, perhaps in the manner described by the "Wind-Compressed Disk" model of Bjorkman \& Cassinelli (1993). We are planning further optical and UV observations of HD 93521 to investigate whether the circumstellar variability is related to the underlying NRP.

\section{Acknowledgements}

AWF thanks the LOC for financial support to attend this Symposium.

\section{References}

Bjorkman, J. E., and Cassinelli, J. P.: 1993, Astrophysical Journal 409, 429

Conti, P. S., and Ebbets, D.: 1977, Astrophysical Journal 213, 438

Fullerton, A. W.: 1990, Ph.D. thesis, University of Toronto

Gies, D. R., and Kullavanijaya, A.: 1988, Astrophysical Journal 326, 813

Howarth, I. D., and Reid, A. H. N.: 1993, Astronomy and Astrophysics, in press

Irvine, N. J.: 1989, A strophysical Journal 337, L33

Massa, D. L.: 1992, in L. Drissen, C. Leitherer, A. Nota, ed(s)., Nonisotropic and Variable Outflows from Stars, ASP: San Francisco, 84

Prinja, R. K., and Howarth, I. D.: 1986, Astrophysical Journal, Supplement Series 61, 357 